\title{
Die Unfallchirurgie in der Chirurgie - Realität und Zielsetzung
}

\author{
G. Hierholzer
}

\section{Einleitung}

Die Chirurgie ist bekanntlich nach einer Vorgeschichte von mehreren Tausend Jahren aus dem handwerklichen Beruf der Barbiere und der Feldschere hervorgegangen. Die Aufgaben beschränkten sich damals weitgehend auf diejenigen eines Wundarztes, er ist der eigentliche Vorfahre des heutigen Chirurgen. Erst im 19.Jahrhundert erlebte die Chirurgie eine breite akademische Anerkennung und erfuhr ihre fachliche Entfaltung in einem Zeitbereich von rund 120 Jahren. Das Bewusstsein um die historischen Zusammenhänge sollte im Chirurgischen Handeln verankert bleiben. Es wird uns immer an die Verantwortung für das Gesamtgebiet erinnern.

In der Zukunft werden weder eine restriktive Bewahrung noch eine übertriebene Spezialisierung die Chirurgie als umfassendes Gebiet erhalten können. Die Mammachirurgie, die Tendenzen der Strumachirurgie, die besorgniserregende Entwicklung der Zuständigkeit für endoskopische Eingriffe, für die Intensivmedizin und für das Rettungswesen sind mahnende Beispiele. Umso gefährlicher ist es, die zweifelsohne notwendige weitere Spezialisierung als Legitimation zur Bildung vom Gesamtgebiet losgelöster Fachgebiete zu verwenden. Im Folgenden wird zu der Frage Stellung genommen, ob der enge Verbund der Unfallchirurgie mit den anderen chirurgischen Arbeitsbereichen weiter begründet ist und erhalten bleiben soll.

\section{Definition}

Für eine einvernehmliche Beurteilung der zukünftigen Entwicklung der Chirurgie ist es wichtig, die Begriffe, die die Chirurgie beschreiben, einheitlich zu verwen-

OP-JOURNAL 2000; 16: 234-236

(c) Georg Thieme Verlag Stuttgart · New York den. In erster Linie betrifft dies die „allgemeine Chirurgie“ als wissenschaftliche und klinische Grundlage unseres Gebietes. Sie beinhaltet die Basiskenntnisse der pathophysiologischen Zusammenhänge, der Operationstechniken und der erarbeiteten Leitlinien. Der Begriff „Allgemeinchirurgie" ist im deutschen Sprachraum dem angelsächsischen entlehnt, er bezieht sich auf den Aufgabenbereich der Viszeralchirurgie.

Die „Allgemeine Chirurgie“ ist die fachliche Basis und damit in ihrer Bedeutung weit mehr. Die einzelnen chirurgischen Arbeitsbereiche haben jeweils keine eigene pathophysiologische und wissenschaftliche Grundlage. An den Stellen, an denen dieses Verständnis fehlt, werden Zeichen einer fachlich falschen Spezialisierung erkennbar. Was nutzen weiterentwickelte Operationsverfahren, die mit besonderem Gerät und einer spezialisierten Technik verbunden sind, wenn gleichzeitig die Regeln der Wundheilung und der Wundbehandlung missachtet werden. Das Beispiel macht deutlich, dass das Verlassen der gemeinsamen fachlichen Basis mit vermeidbaren Misserfolgen verbunden sein kann.

Für die Definition der Chirurgie wurde in den zurückliegenden Jahren immer wieder das Bild eines lebenden Baumes bemüht mit dem Hinweis, der Stamm sei die Viszeralchirurgie. Im Verständnis dieses Bildes muss dann aber die Unfallchirurgie als die historisch belegbare Wurzel des Baumes bezeichnet werden. Nun führt uns das Bemühen, den einzelnen Arbeitsbereichen besondere Wertinhalte zuzuordnen, nicht zu dem erwünschten gemeinsamen Ziel. Es ist ja offenkundig, dass ein Baum weder ohne Wurzel, noch ohne Stamm, aber ebenso wenig ohne Äste lebensfähig ist. Wir sollten uns deshalb auf die Gleichwertigkeit der Strukturen wie auch auf die Bedeutung ihrer inneren Zusammenhänge für die Lebensfähigkeit des ganzen Organismus verstehen.
Die Unfallchirurgie ist wie die anderen chirurgischen Arbeitsbereiche ein unverzichtbarer Bestandteil des Ganzen. Es erscheint unerheblich, ob die derzeitigen Bemühungen um eine Weiterentwicklung der chirurgischen Strukturen in der Zukunft zu einer anderen Nomenklatur der Zuständigkeiten und der heute gültigen Schwerpunktsbezeichnungen führen. Wichtig ist es, dass eine angestrebte Novellierung dem fachlichen $\mathrm{Zu}-$ sammenhalt in der Chirurgie, der Kooperationsbereitschaft und der Gleichberechtigung unter den chirurgischen Kollegen Rechnung trägt. Eine geänderte Nomenklatur von Strukturen und Zuständigkeiten darf also nicht im Widerspruch zum gemeinsamen Ziel stehen. Abspaltungstendenzen lösen weder fachliche Probleme noch kollegiale Fragen.

\section{Chirurgie des Bewegungsapparates}

Es bestehen immer wieder Tendenzen, die Unfallchirurgie mit der Chirurgie des Bewegungsapparates gleichzusetzen. Dieser Versuch, die unfallchirurgische Aufgabenstellung aus dem Zentrum der Chirurgie vertreiben zu wollen, ist fachlich nicht nur unbegründet, er führt zu Ausgrenzungs- und Abspaltungserscheinungen. Diese müssen letztlich dem Gesamtgebiet Schaden zufügen und dem Verständnis der Patienten über den Umfang der chirurgischen Zuständigkeit abträglich sein.

\section{Umfang und Art der unfallchirurgischen Aufgabenstellung}

Gehen wir von rund einer halben Million verletzter Menschen aus, die in Deutschland pro Jahr der stationären Behandlung bedürfen, so umschreibt diese Größenordung nur einen Teil der Gesamtaufgabe, die je nach dem Auftrag an eine Krankenhausabteilung um das 3-5fache höher liegt. Folgeeingriffe, die dem Bereich der Sekundär- und Wiederherstellungschirurgie zuzuordnen sind, die präventive Chirurgie, die Physiotherapie u.a. er- 
weitern die Aufgaben ganz erheblich. Die unfallchirurgische Verantwortung umfasst die diagnostischen und die therapeutischen Maßnahmen nach Verletzungen von der Notversorgung bis zur Beendigung des Heilverfahrens. In diesem $\mathrm{Zu}-$ sammenhang ist auf das berufsgenossenschaftliche Heilverfahren hinzuweisen, das mit Erfolg seit vielen Jahrzehnten in einem ganzheitlichen Verständnis die besten Voraussetzungen dafür bietet, die Anforderungen einer Qualitätssicherung zu erfüllen.

Es gibt aber auch ganz praktische Erfahrungen und Begründungen. Der unfallchirurgische Patient ist in typischer Weise zeitlich unangemeldet und die Aufgabe nicht im organisatorischen Nebenschluss zu bewältigen. Bei den geplanten Operationsprogrammen in chirurgischen Kliniken und Krankenhausabteilungen muss gewährleistet sein, dass der Unfallpatient nach der Aufnahme ohne Zeitverzögerung fachlich kompetent untersucht und behandelt wird. Im Besonderen ist die Koordination der Diagnostik und Therapie zu gewährleisten.

Die Tendenz z.B., die notärztliche Tätigkeit und Erstversorgung dem Aufsichtsund Entscheidungsbereich anderer Fachgebiete zu überlassen, ist in hohem Maße zu beklagen. Es handelt sich hierbei um eine zentrale chirurgische Aufgabe und Verantwortung. Nach dem Bekunden will diese zwar keiner der Kollegen abgeben, es fehlt aber leider zunehmend an der entsprechenden Durchsetzungskraft, sie umzusetzen. Die Zuständigkeit anderen Fachgebieten zuzuordnen, ist ebenso falsch wie die aktuelle Tendenz, z. B. die Schmerzbehandlung aus der chirurgischen Therapie herauszunehmen und dafür ein neues Gebiet zu schaffen.

Der Unfallchirurg wird selbstverständlich die Kompetenz der anderen chirurgischen Arbeitsbereiche mit einbeziehen und somit keine allumfassende Zuständigkeit beanspruchen. Für die Koordination der Maßnahmen nach einer Mehrfachverletzung zuständig zu sein, bedeutet also nicht, als Unfallchirurg für jede Form der Organverletzung therapeutisch allein kompetent sein zu wollen. Die Frage, bis zu welchem Schweregrad der Unfallchirurg z.B. die Höhlenverletzungen versorgen soll, ist nicht durch eine Form der fachlichen Abgrenzung, sondern durch organisatorische Richtlinien und durch die Gewährleistung der $\mathrm{Zu}$ sammenarbeit unter den fachlichen Schwerpunkten zu beantworten. Es ent- scheiden somit die Art und das Ausmaß der Verletzung darüber, welcher Kollege operiert und welcher assistiert. Diese innerhalb einer Klinik unter den speziellen Arbeitsbereichen zu lösende Aufgabe berührt nicht das gesamtchirurgische Behandlungskonzept, das der Erwartung und dem Vertrauen des Patienten entsprechen soll.

Die Auffassung, diese Konzeption sei unrealistisch, teile ich nicht. Vielfältige Rundreisen in den letzten beiden Jahren in den östlichen Bundesländern haben gezeigt, dass es besonders hier zahlreiche Kliniken und Krankenhausabteilungen gibt, die dieses Konzept kollegial und erfolgreich praktizieren. Die Nomenklatur und die fachlichen Zuständigkeiten sind dabei keineswegs einheitlich, dies widerspricht aber nicht der Aussage.

Bei der Diskussion sollte man immer wieder auf das Beispiel einer Anwaltskanzlei hinweisen, in der Arbeitsteilungen dem Ziel dienen, den fachlichen Anforderungen bestmöglich gerecht werden zu können. In einer derartigen Sozietät wird kein Kollege dem anderen absprechen, ebenfalls Anwalt und gleichberechtigter Partner zu sein. Muss es eine Illusion sein, darauf zu hoffen, dass wir Chirurgen dazu ebenfalls fähig sind?

\section{Forschung, Lehre, Weiterbildungsfragen}

Aus dem fachlichen Verständnis der chirurgischen Verantwortung lassen sich unschwer die Richtlinien für die Forschung und die Lehre ableiten. Wie für die anderen chirurgischen Arbeitsbereiche muss es in Relation zu der klinischen Gesamtaufgabe für die Unfallchirurgie ausreichende Forschungseinrichtungen geben. Als Konsequenz daraus sind an den Universitäten entsprechende Lehrstühle vorzuhalten. Es bedarf wohl auch keiner weiteren Begründung, dass die unfallchirurgische Weiterbildung nicht durch andere fachliche Schwerpunkte wahrgenommen werden kann. An manchen Universitäten wird diesen Anforderungen mit dem vermeintlichen Argument einer Kosteneinsparung nicht entsprochen. Dabei verkennt man offensichtlich die für diese Universitäten und für das Gesamtgebiet der Chirurgie entstehenden negativen Auswirkungen.

Die Weiterbildung, in die auch die außeruniversitären Krankenhäuser einbezogen sind, erfordert ebenfalls eine chirurgische Krankenhausstruktur mit innerem Ver- bund. Im Assistentenbereich sollte der Verbund mit einem abgestimmten Weiterbildungsplan und z.B. mit einem gemeinsamen Bereitschaftsdienst verwirklicht sein. In den speziellen chirurgischen Abteilungen sind die Weiterbildung wie die Therapie einer eigenständigen Verantwortung der leitenden Ärzte zu unterstellen. Die Verbundstruktur wird fachlich durch einen getrennten Hintergrunddienst abgesichert.

\section{Chirurgie und Qualitätssicherung}

Zahlreiche Beispiele zeigen, dass diejenigen Kliniken und Krankenhäuser, die der qualitätssichernden Anforderung nicht entsprechen, ihre Lebensfähigkeit verlieren. Im Einzelnen wäre es tragisch, zu der Erkenntnis erst über den Weg der zunehmenden Haftungsverfahren zu kommen. Es wird die Meinung vertreten, der unstrukturierte chirurgische Krankenhausbereich sei mit gleichem fachlichen Qualitätsanspruch kostengünstiger $\mathrm{zu}$ führen. Die Analyse der fachlichen Arbeit der Belegungsrate von Abteilungen und der Kosten haben ein derartiges Argumentieren widerlegt.

In einem kleineren Krankenhaus, in dem eine fachliche Strukturierung nicht $\mathrm{zu}$ realisieren ist, bedarf es der Beschränkung auf eine objektivierbare chirurgische Zuständigkeit und für die darüber hinausgehenden Anforderungen der $\mathrm{Zu}$ sammenarbeit mit einem erreichbaren Zentrum. In diesem Verständnis ist auch eine unstrukturierte chirurgische Abteilung z.B. in einem ländlichen Gebiet vertretbar und ggf. notwendig. Ebenso kann ein fachlicher Verbund unmittelbar benachbarter Krankenhäuser praktiziert werden. Es gibt also verschiedene organisatorische Möglichkeiten, dem Gebot der qualitätssichernden Zusammenarbeit der Chirurgen Rechnung zu tragen.

\section{Struktur chirurgischer Kliniken und Krankenhausabteilungen}

Für die Chirurgie ist folgende Richtlinie vorzuschlagen:

1. In der chirurgischen Abteilung eines Krankenhauses der Regelversorgung sollte eine unfallchirurgische Dauerposition geschaffen werden. Umfasst der chirurgische Arbeitsbereich mehr als $100-120$ Betten, so ist nach den o.g. Überlegungen eine Aufgabenteilung sinnvoll. Die Anforderungen an den administrativ übergeordneten Ärztlichen Direktor einer Klinik ergeben sich aus den erforderlichen Persönlich- 
keitsmerkmalen, der Kompetenz und der Integrationsfähigkeit. Die Position kann somit nicht grundsätzlich an einen der speziellen Arbeitsbereiche gebunden sein.

2. An den Universitäten sind unfallchirurgische Lehrstühle zur Sicherstellung von Forschung, Lehre, Weiterentwicklung der Therapie und für die Weiterbildung unabdingbar.

3. Die Ermächtigung zur Therapie und zur klinischen Weiterbildung hat sich an Kriterien zu orientieren, die dem jeweiligen Stand der Anforderungen an die Qualitätssicherung entsprechen.

\section{Anmerkungen zum Nachbargebiet Orthopädie}

Es ist nicht die Aufgabe der Unfallchirurgen, über das Gebiet Orthopädie zu befinden. Da die Orthopäden im Benehmen zur Unfallchirurgie aber selbst die $\mathrm{Zu}$ kunftsfrage aufgeworfen haben, sollte man die aufgenommenen Gespräche konstruktiv führen. Aus der Sicht des derzeit gültigen Modells der vier chirurgischen Schwerpunkte ist die Integration der chirurgisch tätigen Orthopäden an die Voraussetzung gebunden, sich in die chirurgische Struktur einzubinden, d.h. wieder unter das Dach der Chirurgie zu treten. Sie sind willkommen.

Die zustimmenden und ablehnenden Argumente einer Reintegration werden seit längerem diskutiert. Ablehnende Überlegungen verlieren derzeit an politischer Relevanz. Eine entscheidende Tendenz für die zukünftige Entwicklung ergibt sich aus der internationalen bzw. europäischen Regelung. In diesem Zusammenhang hat die deutsche Weiterbildungsordnung einen Harmonisierungsbedarf mit den europäischen Strukturen.

Die Diskussion ging in den zurückliegenden Jahren oft von der Prämisse aus, europäisches Recht werde das nationale Recht nicht brechen. In der Praxis hat sich diese Feststellung nicht bewahrheitet. Im Allgemeinen orientieren sich nationale Neuregelungen sehr wohl am europäischen Recht in dem erkennbaren Bemühen, zu einer Harmonisierung zu kommen. Es gibt einen weiteren Grund für den Handlungsbedarf. In einigen Bereichen in Deutschland ist die Tendenz erkennbar, chirurgisch-traumatologische und orthopädisch-chirurgische Abteilungen zusammenzulegen. Diese durch die Politik in Gang gesetzte Entwicklung darf von uns nicht übersehen werden.

\section{Schlussfolgerung}

Das Gesamtgebiet Chirurgie leitet sich aus seiner historischen Entwicklung und aus einem umfassenden Verständnis des Behandlungsauftrages ab. Die immer komplexer werdende Diagnostik und Therapie unterstreichen die Forderung nach einem engen Verbund der sich spezialisierenden Arbeitsbereiche.

An der Realität der umfangreichen unfallchirurgischen Aufgabe besteht kein Zweifel. Die Behandlung der Folgen eines Traumas ist aber ein unverzichtbarer Bestandteil und Inhalt der chirurgischen Gesamtaufgabe.

Die Strukturierung der Chirurgie in partnerschaftlich gleichberechtigte Arbeitsbereiche erfüllt die Anforderungen an Statuten, die in die Zukunft gerichtet sind. Nicht ausbleibende fachliche und kollegiale Probleme sind unter den Chirurgen zu regeln.

\section{Prof. Dr. med G. Hierholzer}

Radolfzeller Str. 109

78476 Allensbach 